\title{
PERFIL CLÍNICO E EPIDEMIOLÓGICO DO LÍQUEN PLANO BUCAL: UM ESTUDO DESCRITIVO
}

\author{
Bruno Oliveira Queiroz'; Valéria Souza Freitas²; Alessandra Laís Pinho Valente \\ Pires $^{3}$ e Juliana Araújo da Silva Simoura ${ }^{4}$ \\ 1. Bolsista PROBIC/UEFS, Graduando em Odontologia, Universidade Estadual de Feira de Santana, e-mail: bruno- \\ queiroz2008@hotmail.com \\ 2. Orientadora, Departamento de Saúde, Universidade Estadual de Feira de Santana, e-mail: valeria.souza.freitas@gmail.com \\ 3. Participante do Núcleo de Câncer Oral/NUCAO, Departamento de Saúde, Universidade Estadual de Feira de Santana, e- \\ mail: lecavalent@hotmail.com \\ 4. Participante do Núcleo de Câncer Oral/NUCAO, Departamento de Nome, Universidade Estadual de Feira de Santana, e- \\ mail: julianasimoura.uefs@gmail.com
}

PALAVRAS-CHAVE: líquen plano bucal; doenças da boca; epidemiologia.

\section{INTRODUÇÃO}

O Líquen plano bucal (LPB) é uma doença inflamatória crônica que acomete a mucosa bucal (Randazzo et al., 2005). Estudos evidenciam que o LPB usualmente acomete de 0,5 a 2\% da população em geral (Sugerman \& Savage, 2002; Fernándéz-Gonzáles et al., 2010), do com predileção por indivíduos do sexo feminino (Ingafou et al., 2006; Bermejo-fenoll et al., 2009; Carbone et al., 2009), entre a quarta e a sexta década de vida (Farhi \& Dupin, 2010).

O LPB apresenta uma grande diversidade de aspectos clínicos (reticular, erosiva, bolhosa, atrófica, pápula e placa), podendo acometer qualquer região da cavidade bucal, incluindo principalmente a mucosa jugal, o dorso da língua, o vermelhão do lábio e a gengiva (Mollaoglu, 2000). A descrição detalhada das características clínicas da lesão, da distribuição anatômica e a presença de lesões cutâneas auxiliam o diagnóstico clínico. Entretanto, a análise histopatológica é fundamental para obter o diagnóstico conclusivo do LPB (Mollaoglu, 2000; Kelsch \& Edwards, 2002). Deste modo, este estudo tem por finalidade descrever o perfil clínico-epidemiológico dos casos de LPB diagnosticados no Centro de Referência de Lesões Bucais da Universidade Estadual de Feira de Santana (CRLB-UEFS), no período de 2010 a 2017.

\section{MATERIAL E MÉTODOS}

Trata-se de um estudo observacional do tipo descritivo, retrospectivo, baseado em dados secundários dos registros de prontuários clínicos dos indivíduos diagnosticados com LPB atendidos no CRLB-UEFS no período de 2010 a 2017.

A população do estudo foi constituída por todos os casos com diagnóstico clínico e histopatológico de LPB, pertencentes ao arquivo do CRLB-UEFS no referido período. A coleta de dados foi realizada com um uso de uma ficha específica contendo informações sobre as variáveis sócio demográficas (idade, sexo, cor da pele, escolaridade, ocupação e situação conjugal); condições sistêmicas e de saúde (presença de menopausa, doença sistêmica); hábitos de vida (consumo de tabaco e bebidas alcoólicas) e dados clínicos referentes à lesão (forma clínica e localização anatômica).

Os dados obtidos foram analisados, descritivamente, com o uso do programa estatístico Statistical Package for the Social Sciences (SPSS) na versão 17.0 (SPSS Inc., Chicago, IL, USA). O presente estudo foi aprovado pelo Comitê de Ética em Pesquisa em Seres Humanos da UEFS, com parecer n ${ }^{\circ}$ 114.132 e CAAE: 5590612.7.0000.0053. 


\section{RESULTADOS E DISCUSSÃO}

Foram diagnosticados clínico/histopatologicamente 18 casos de LPB, no período de 2010 a 2017. A média de idade dos indivíduos foi de $50( \pm 14,95)$ anos, com maior número de casos com idade acima dos 40 anos $(66,7 \%)$.

A maioria dos indivíduos era do sexo feminino (72,2\%), de cor da pele preta, $(58,8 \%)$, com escolaridade no ensino médio (38,9\%), sendo que $50 \%$ destes apresentavam-se com situação conjugal com e sem companheiro. A análise da situação de trabalho demonstra que $66,7 \%$ dos investigados eram ocupacionalmente ativos.

O LPB acomete com mais frequência indivíduos do sexo feminino (Mostafa \& Ahmed, 2015) entre a quarta e a sexta décadas de vida (Zanatta et al., 2011; Villanova et al., 2012; Mostafa \& Ahmed, 2015), estando os achados do presente estudo de acordo com a literatura. Com relação à variável cor da pele, os nossos achados contrapõem os resultados de alguns autores que encontraram predileção da doença em indivíduos de cor da pele branca (Burkhart et al., 1996; Sousa \& Rosa, 2008; Vilanova et al., 2012; Filiu et al., 2013). Esta discordância pode ser justificada pela miscigenação da população da região da Bahia, com maior presença de indivíduos pardos e pretos (IBGE, 2010).

Dentre os fatores sóciodemográficos investigados, observou-se que o maior percentual de indivíduos acometidos (38,9\%) apresentava escolaridade no nível médio e, 66,7\% eram ocupacionalmente ativos. Escolaridade e situação ocupacional não têm sido reportadas como fatores que compõem a possível rede de causalidade do LPB. Entretanto, tais variáveis têm sido apontadas como contribuintes importantes para a exacerbação da ansiedade, depressão e estresse, como reportado por alguns autores (Pokupec et al., 2009; Girard et al., 2011).

Quanto às condições de saúde e estilo de vida dos indivíduos investigados, 55,6\% das mulheres relataram não estar na fase de menopausa. Com relação ao estilo de vida, o uso do tabaco ocorreu em apenas $27,8 \%$ e o consumo de bebidas alcoólicas em $44,4 \%$ da população do presente estudo. Resultados semelhantes foram encontrados por outros autores, apesar do não estabelecimento da relação causal entre estes hábitos e o LPB (Zanatta et al., 2011).

Considerando a totalidade de indivíduos do nosso estudo, 61,1\% relataram alguma doença sistêmica, sendo a hipertensão arterial $(27,8 \%)$ a mais frequente, seguida da hipertensão e diabetes concomitantemente $(16,7 \%)$. De acordo com os resultados encontrados por Vilanova et al. (2012), em um estudo envolvendo 52 casos de LPB, a gastrite foi a condição mais frequente na amostra estudada, correspondendo a 17,30\% dos casos investigados, seguida da hipertensão $(15,38 \%)$ e diabetes mellitus $(3,84 \%)$. A prevalência de hipertensão arterial e diabetes mellitus em indivíduos com LPB têm sido observada, porém a influência que estas alterações sistêmicas exercem na etiologia e no curso clínico da doença ainda não foi esclarecida (Fang et al., 2009). Além disso, o uso de medicamentos para o controle de hipertensão e diabetes pode estar relacionado ao surgimento de lesões liquenóides orais (Barbosa et al., 2015), o que indica a necessidade de um correto diagnóstico para esta lesão.

A forma clínica da doença mais observada foi a reticular, por vezes envolvendo mais de um sítio anatômico, estando este achado de acordo com outros estudos (Dorta et al., 2001; Canto et al., 2010; Fraga et al., 2011). Na maioria dos casos esta forma clínica não provoca sintomas e se apresenta geralmente de forma bilateral em região posterior de mucosa jugal sob a forma de um rendilhado de estrias brancas que se entrecruzam, conhecidas como estrias de Wickham. No entanto, esses achados contrapõem os de Fenoll et al. (2010) e Gümrü (2013), que encontraram uma predominância da forma erosiva, a segunda mais frequente do nosso estudo. Barbosa et al. (2015) acreditam que devido às lesões erosivas serem sintomáticas este fato pode contribuir para um aumento da sua frequência devido a maior 
procura dos indivíduos com esta forma clínica aos serviços de saúde para o controle dos sintomas.

Com relação à localização da lesão, a mucosa jugal foi a mais acometida corroborando com os dados da literatura (Sugerman \& Savage, 2002; Souza \& Rosa, 2005; Zanatta et al., 2011; Vilanova et al., 2012; Filiu et al., 2013). De acordo com alguns autores, esta localização anatômica é a de maior frequência para o LPB reticular (Vilanova et al., 2012; Budimir et al., 2014), a forma clínica mais observada do presente estudo.

Diante do exposto ressalta-se também a importância de analisar o perfil epidemiológico, a história da doença, suas características clínicas e histopatológicas para o diagnóstico conclusivo do LPB. Os achados desta investigação não permitem inferir causalidade, devido às limitações do delineamento descritivo. Assim, para um melhor esclarecimento dos fatores etiológicos relacionados ao LPB novos estudos longitudinais devem ser realizados.

\section{CONCLUSÃO}

O perfil clínico epidemiológico dos indivíduos com LPB revela que a maioria eram mulheres, com idade superior a 40 anos, pretos ou pardos, com escolaridade no ensino médio e ocupacionalmente ativas. A forma clínica reticular foi a mais predominantemente, localizando-se geralmente na mucosa jugal. $O$ presente estudo deve contribuir para um melhor entendimento do perfil clínico epidemiológico dos indivíduos com LPB, atendidos no CRLBUEFS no período investigado, podendo seus resultados se constituir em uma importante ferramenta para o planejamento de ações para o controle desta lesão, bem como para elaboração de protocolos de tratamento mais adequados.

\section{REFERÊNCIAS}

BARBOSA, et al. 2015. Factors associated with clinical characteristics and symptoms in a case series of oral lichen planus. International Journal of Dermatology, 54: e1-e6.

BERMEJO-FENOLL, A., et al. 2009. Premalignant nature of oral lichen planus. A retrospective study of 550 oral lichen planus patients from south-eastern Spain. Oral Oncology, 45 (8): 54-56.

BURKHART, N.W., BURKER, E.J., BURKES, E. J., WOLFE, L. 1996. Assessing the characteristics of patients with oral lichen planus. JADA, 127: 648-661.

CANTO, A. M., MÜLLER, H., FREITAS, R. R., SANTOS, P. S. S. 2010. Líquen plano oral (LPO): diagnóstico clínico e complementar. An. Bras. Dermatol, 85 (5): 669-675.

CARBONE, M., et al. 2009. Course of oral lichen planus: a retrospective study of 808 northern Italian patients. Oral Diseases, 15: 235-243.

CENSO DEMOGRÁFICO. 2010 [online]. Homepage: http://www.ibge.gov.br/home/estatistica/populacao/censo2010/caracteristicas_religiao_de ficiencia/caracteristicas_religiao_deficiencia_tab_uf_xls.shtm.

DORTA, R. G., et al. 2001. Gingival erosive lichen planus: case report. Brazilian dentistry journal, 29 (1): 63-66.

FANG, M., et al. 2009. Malignant transformation of oral lichen planus: A retrospective study of 23 cases. Quintessence International, 40 (3): 235-224.

FARHI, D., DUPIN, N. 2010. Pathophysiology, etiologic factors, and clinical management of oral lichen planus, part I: facts and controversies. Clinics in Dermatology, 28: 100-108.

FENOLL, A. B., et al. 2010. A retrospective clinicopathological study of 550 patients with oral lichen planus in south-eastern Spain. J. Oral Pathol. Med., 39: 491-499.

FRAGA, H. F. et al. 2011. A importância do diagnóstico do líquen plano bucal. J. Health Sci. Inst., 29 (1): 27-30. 
GIRARDI, C., et al. 2011. Salivary cortisol and dehydroepiandrosterones (DHEA) levels, psychological factors in patients with oral lichen planus. Archives Oral Biology, 56 (9): 864-868.

GÜMRÜ, B. 2013. A retrospective study of 370 patients with oral lichen planus in Turkey. Med. Oral Patol. Oral Cir. Bucal, 18 (3): 427-432.

MOLLAOGLU, N. 2000. Oral lichen planus: a review. British Journal of Oral and Maxillofacial Surgery, 38 (4): 370-377.

PETERSEN, P.E. 2009. Oral cancer prevention and control-The approach of the World Health Organization. Oral Oncology, 45 (4): 454-460.

POKUPEC, J. S., GRUDEN, V., GRUDEN JR, V. 2009. Lichen ruber planus as a psychiatric problem. Psychiatria Danubina, 21 (4): 514-516.

RANDAZZO, A.R., AMORMINO, S.A.F., MARTINS, C.R. 2005. Líquen plano bucal e hepatite C: revisão de literatura. Rev. de Clín. Pesq. Odontol., 1 (3).

SOUZA, F.A.C.G., ROSA, L.E.B. 2008. Líquen plano bucal: considerações clínicas e histopatológicas. Rev. Bras. Otorrinolaringol., 74 (2): 284-92.

SUGERMAN, P. B., SAVAGE, N. W. 2002. Oral lichen planus: causes, diagnosis and management. Australian Dental Journal, 47 (4): 290-297.

SUGERMAN, P.B., SAVAGE, N.W. 2002. Oral lichen planus: causes, diagnosis and management. Australian Dental Journal, 47 (4): 290-297.

VILANOVA, L. S. R., DANIELli, J., PEDROSO, L., SILVA, M. A. G. S. 2012. Perfil epidemiológico de portadores de líquen plano oral atendidos no Centro Goiano de Doenças da Boca (CGDB) - 12 anos de experiência. Rev. Odontol. Bras. Central, 21 (59): 226-229.

ZANATTA, R. F., ROSA, R. R., DE PAUlO, L. F. B., DURIGUETTO JUNIOR, A. F. 2011. Prevalência das lesões de líquen plano atendidos no ambulatório da Unidade de Diagnóstico Estomatológico UFU no período de 1997 a 2008. Rev. Horiz. Cient. 5 (1). 